\title{
Meta-analysis of synovial fluid polymerase chain reaction for diagnosing periprosthetic hip and knee infection
}

\author{
Cheng $\mathrm{Li}^{1,2^{*}}\left(\mathbb{0}, \mathrm{Hao} \mathrm{Li}^{3}\right.$, Xue Yang ${ }^{3}$, Fang-Zheng Zhu ${ }^{3}$, Chi Xu ${ }^{3}$ and Andrej Trampuz ${ }^{2}$
}

\begin{abstract}
Background and objective: The purpose of this study was to estimate the diagnostic performance of synovial fluid polymerase chain reaction (PCR) in periprosthetic hip and knee infection, and whether synovial fluid PCR has greater diagnostic significance than conventional methods.

Methods: The literature databases PubMed, Scopus, and the Web of Science were searched for English articles describing periprosthetic joint infection (PJI) diagnosis by synovial fluid PCR. Articles were limited to the period between January 1990 and December 2019. Subsequently, conventional methods that were used on at least two occasions were included for further analysis. Data analysis was performed using the Meta-DiSc and Stata software.

Results: Eleven studies with 1360 cases were included in the meta-analysis. The pooled sensitivity, specificity, and diagnostic odds ratio (DOR) of synovial fluid PCR were 0.70 ( $95 \% \mathrm{Cl} 0.66-0.74), 0.92$ ( $95 \% \mathrm{Cl} 0.90-0.93$ ), and 37.4 (95\% Cl 17.77-78.74), respectively.

Conclusions: Synovial fluid PCR provides an effective tool for rapid diagnosis of PJI, and also in the early stages of culture-negative bacterial infections.
\end{abstract}

Keywords: Meta-analysis, Diagnosis, Arthroplasty, Periprosthetic joint infection, Synovial fluid, Polymerase chain reaction

\section{Background}

Periprosthetic joint infection (PJI) is one of the most severe complication following hip or knee arthroplasty, with high morbidity, mortality and costs [1-5]. However, its diagnosis remains a challenge in the management of PJI. To date, there is no test available that rapidly and accurately diagnoses infection with a sensitivity and specificity of $100 \%$ [6]. A mixture of several diagnostic methods or diagnostic definition that guide the diagnosis of PJI is currently the most common approach used [7].

*Correspondence: cheng.li@charite.de

${ }^{2}$ Center for Musculoskeletal Surgery (CMSC), Charité-Universitätsmedizin Berlin, Berlin, Germany

Full list of author information is available at the end of the article
Joint aspiration is an invasive diagnostic method and often used as the first step in suspected PJI cases. Currently, various synovial fluid tests have been applied in the clinical diagnosis of PJI $[8,9]$. Non-microbiological analysis of the synovial fluid white cell count and polymorphonuclear (PMN) leukocytes, alpha-defensin, leukocyte esterase (LE), and C-reactive protein (CRP) were included in the new definition of PJI from the Musculoskeletal Infection Society (MSIS) guideline of 2018 [10]. Although these tests are of high diagnostic value for PJI cases, they are unable to identify the causative pathogen. Conventional synovial fluid culture still appears to be an irreplaceable diagnostic approach for the detection of microorganisms. In recent years, polymerase chain reaction (PCR) was also used in the diagnosis of PJI. A number of reports found that synovial fluid PCR could rapidly 
detect rare bacterial infections, which is in contrast to conventional synovial fluid culture $[11,12]$. However, it remains unknown whether better diagnostic results are observed using PCR compared to tissue culture, with several conclusions arguable [13, 14]. Although a metaanalysis of PCR for diagnosis of PJI has been previously performed using results combined from synovial fluid, periprosthetic tissue, and sonicate fluid samples [15], the diagnostic value of synovial fluid PCR in the periprosthetic hip and knee remains unclear.

The aim of the present meta-analysis was to assess the diagnostic accuracy of synovial fluid PCR in periprosthetic hip and knee infection. Furthermore, we examined whether synovial fluid PCR has a better diagnostic value compared to conventional methods, especially synovial fluid culture.

\section{Methods}

\section{Search strategy}

A systematic literature search of the electronic databases of Web of Science, PubMed, and Scopus was performed for manuscripts of English language between January 1990 and December 2019. The following medical subject headings (MeSH) or text keywords were used: "arthroplasty or joint prosthesis or joint replacement or periprosthetic joint or prosthetic joint", "infection or infectious or infected", "synovial fluid", and "PCR or polymerase chain reaction".

Two authors (LC and LH) independently selected research papers according to the following inclusion criteria: (1) human studies related to synovial fluid PCR in periprosthetic hip or knee infection; (2) clear description of the diagnosis standard of PJI; and (3) values on truepositive (TP), false-positive (FP), true-negative (TN), and false-negative (FN) were provided or could be computed.

The combined database was imported to EndNote X7 (Thomson Reuters, New York, NY, USA, 2013). All relevant publications on the synovial fluid PCR method in detecting periprosthetic hip or knee joint infection and their reference list were reviewed. Furthermore, included studies from two previous meta-analyses of synovial PCR were reviewed $[15,16]$. In addition, other diagnostic methods that appeared accumulatively on at least two occasions in all studies were collected. Diagnostic classification values of TP, FP, TN, and FN were included and further compared with synovial fluid PCR.

\section{Data extraction and study quality assessment}

Characteristics of the included studies were collected by two reviewers independently and assessed subsequently by a third reviewer. The following information was extracted: first author, year of publication, country, study design, number of total cases, infection site, acquisition time, diagnostic criteria, type of PCR, target gene, antimicrobial use before specimen collection, and diagnostic sensitivity and specificity. The quality of all identified synovial fluid studies was evaluated using the Quality Assessment of Diagnostic Accuracy Studies (QUADAS-2) guidelines.

\section{Statistical analysis}

To estimate the diagnostic value of synovial fluid PCR for PJI detection, all statistical analyses were performed using Meta-DiSc (version 1.4, Unit of Clinical Biostatistics team, Madrid, Spain) and Stata software (version 14.0, StataCorp, College Station, TX, USA). $I^{2}$ was calculated to evaluate heterogeneity among the studies. If $I^{2}>50 \%$, the random-effects model was used. Metaregression analyses were performed to further assess the potential source of heterogeneity, such as type of prosthesis, number of patients, acquisition time, antibiotic use, sample condition, diagnostic standard, and target gene. Deeks' funnel plot asymmetry test was used to evaluate potential publication bias.

\section{Results \\ Search results}

Of the identified 145 primary articles, two records were extracted from the reference list of synovial fluid PCRrelated studies [17, 18]. Fifty-one studies were excluded due to duplication reasons. A further 83 were excluded after further reviewing the title, abstract, and full text. Finally, a total of 11 studies were considered suitable for meta-analysis (Fig. 1) [13, 14, 17-25]. Characteristics of the studies included are summarized in Table 1. Among these studies, five diagnostic methods could be compared with synovial fluid PCR. The QUADAS-2 quality assessments for the included studies are shown in Fig. 2.

\section{Diagnostic accuracy}

The random-effects model was used to examine study heterogeneity, which was found for the sensitivity $\left(I^{2}=73.9 \%\right)$, specificity $\left(I^{2}=86.0 \%\right)$, positive likelihood ratio (PLR) $\left(I^{2}=77.4 \%\right)$, negative likelihood ratio (NLR) $\left(I^{2}=62.2 \%\right)$, and diagnostic odds ratio (DOR) $\left(I^{2}=66.4 \%\right)$. The pooled sensitivity, specificity, PLR, NLR, and DOR estimates for the diagnosis of PJI using synovial fluid PCR were 0.70 (95\% CI 0.66-0.74), 0.92 (95\% CI $0.90-0.93$ ), 9.09 (95\% CI 5.28-15.67), 0.32 (95\% CI 0.24-0.42), and 37.4 (95\% CI 17.77-78.74), respectively (Figs. 3, 4, 5, 6, 7). The summary receiver operating characteristic (SROC) plot showed the summary sensitivity and specificity and the 95\% confidence and prediction regions, with an area under the curve (AUC) of 0.9252 (standard error of 0.0195; Fig. 8). 


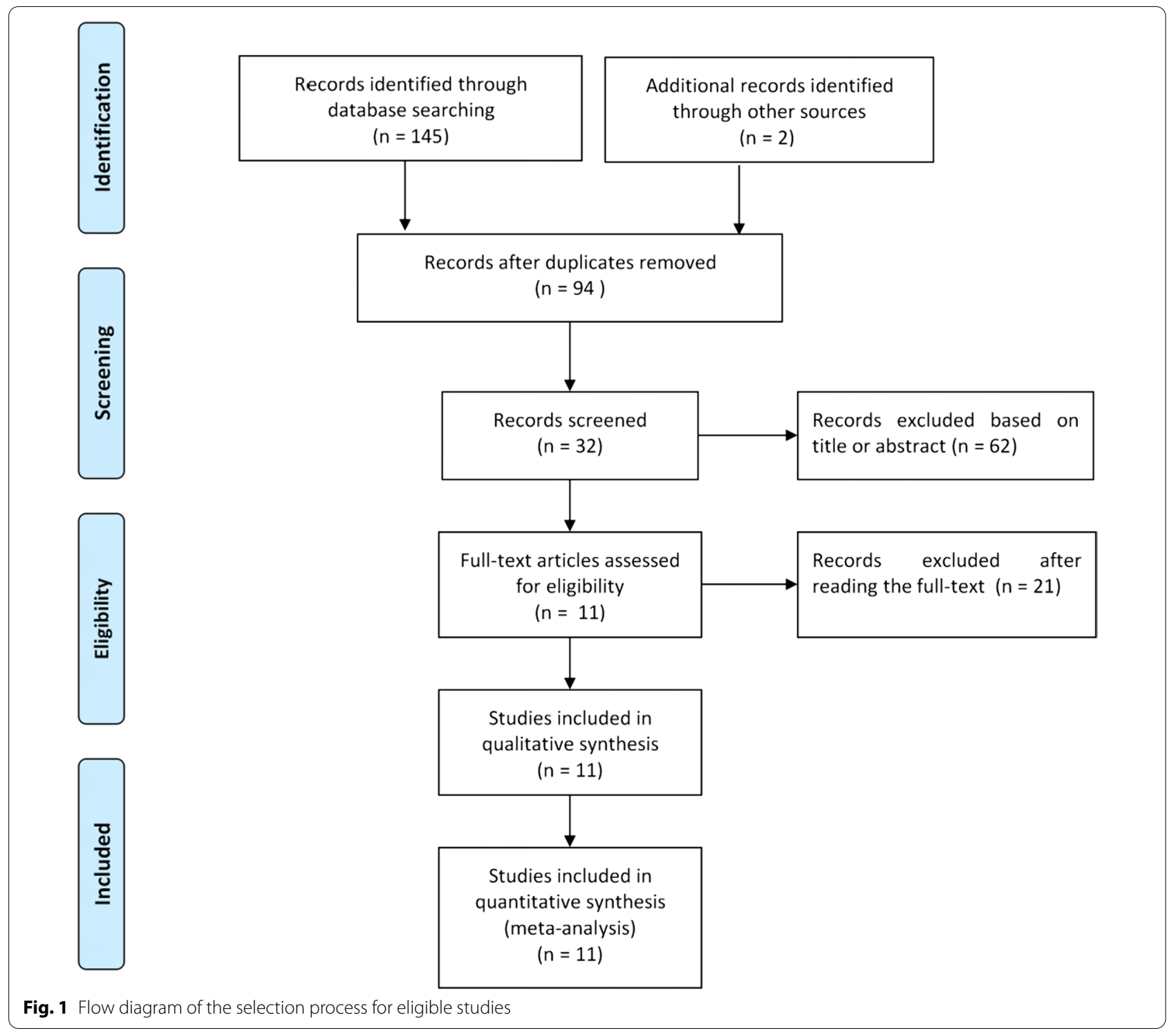

Synovial fluid PCR was shown to have a better sensitivity and specificity than preoperative examination of CRP and erythrocyte sedimentation rate (ESR). Similar sensitivities were observed for conventional synovial fluid culture (70\%) and PCR (69\%). However, the specificity of synovial fluid PCR was lower than that of synovial fluid culture (91\% vs. $98 \%$, respectively). Intraoperative histology, periprosthetic tissue culture, and sonicate fluid demonstrated better sensitivity and specificity than synovial fluid PCR. Details of the diagnostic values of synovial fluid PCR and other preoperative and intraoperative examination are shown in Table 2.

\section{Meta-regression analysis}

Meta-regression analysis was performed in the group of diagnostic standards, acquisition time, number of patients, antibiotic use, and target gene (Table 3). Results showed that the most likely sources of heterogeneity were acquisition time and antibiotic treatment $(P<0.001)$. Compared with the diagnostic standards of MSIS, International Consensus Meeting (ICM), or European Bone and Joint Infection Society (EBJIS), other diagnostic criteria had a higher sensitivity of 0.85 (95\% CI 0.72-0.98; $P<0.05$ ). A small sample size $(<100)$ was observed to have a higher sensitivity of 0.81 (95\% CI 0.71-0.90) compared to studies including 


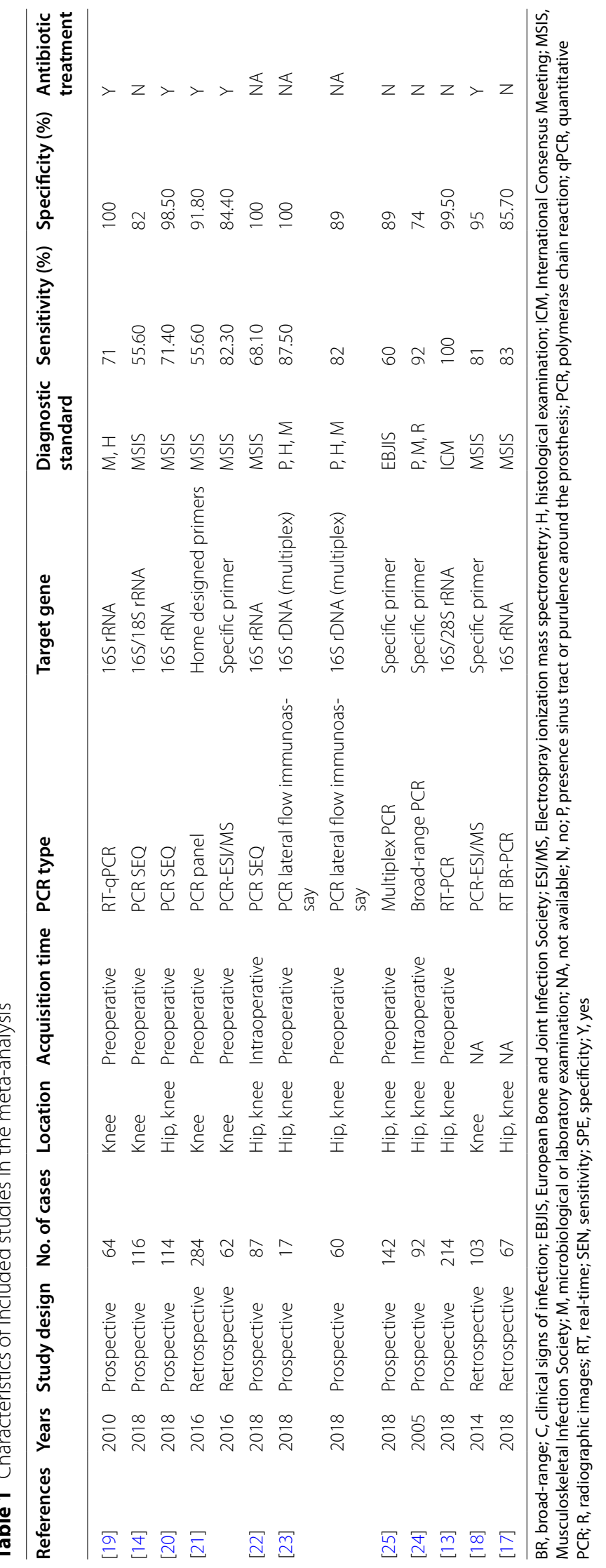




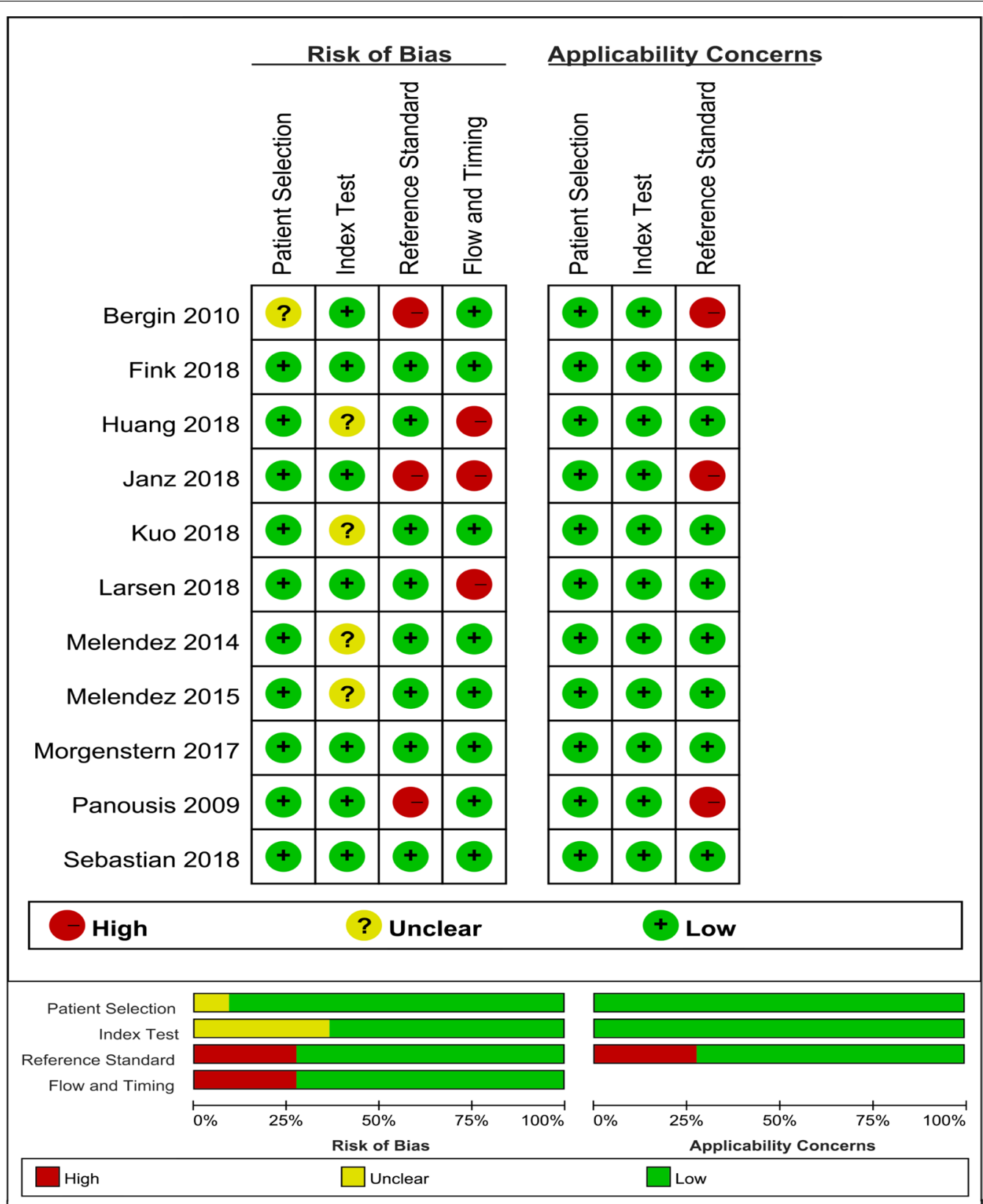

Fig. 2 Methodological quality assessment of the included studies. Cl, confidence interval; AUC, Area under the curve; DOR, Diagnostic odds ratio; CRP, C-reactive protein; IL-6, Interleukin-6; LE, Leukocyte esterase; PCR, Polymerase chain reaction; PMN, Polymorphonuclear leukocytes; WBC, White blood cell

more than 100 patients with a sensitivity of 0.71 (95\% CI 0.59-0.83) $(P=0.01)$.

\section{Assessment of publication bias}

Deeks' funnel plot analysis did not identify a potential publication bias for synovial fluid PCR $(P=0.41$; Fig. 9).

\section{Discussion}

The present meta-analysis showed that the pooled sensitivity and specificity of synovial fluid PCR are $70 \%$ and $92 \%$, respectively. The AUC value of the SROC was 0.9252. These results suggest that synovial fluid PCR could be used for the diagnosis of infection after hip and knee arthroplasty. The first meta-analysis of the use of PCR in the diagnosis of PJI presented the sensitivity and 


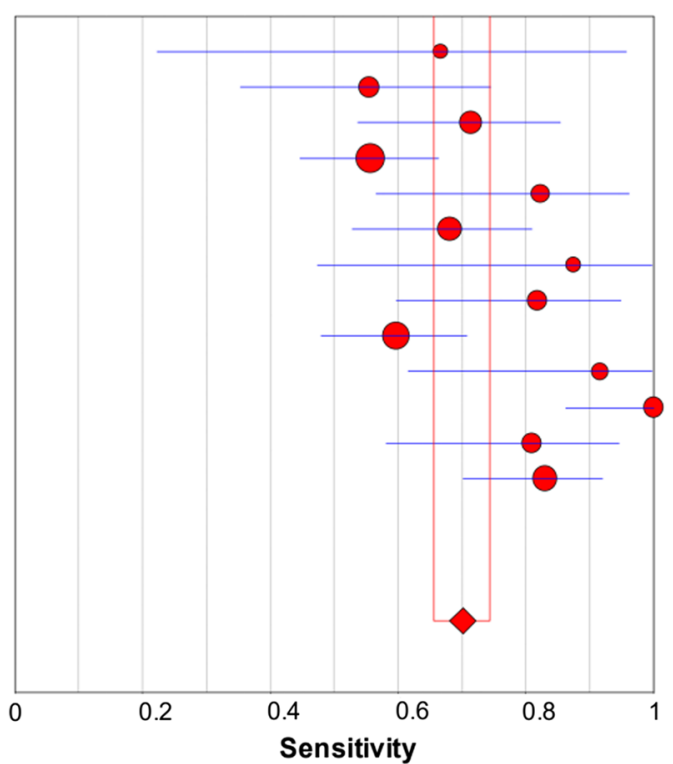

Bergin

Fink

Larsen

Melendez

Melendez

Sebastian

Janz

Janz

Morgenstern

Panousis

Kuo

Melendez

Huang

\section{Sensitivity $(95 \% \mathrm{Cl})$}

$0.67 \quad(0.22-0.96)$

$0.56 \quad(0.35-0.75)$

$0.71 \quad(0.54-0.85)$

$0.56 \quad(0.45-0.66)$

$0.82 \quad(0.57-0.96)$

$0.68 \quad(0.53-0.81)$

$0.88 \quad(0.47-1.00)$

$0.82 \quad(0.60-0.95)$

$0.60 \quad(0.48-0.71)$

$0.92 \quad(0.62-1.00)$

$1.00 \quad(0.86-1.00)$

$0.81 \quad(0.58-0.95)$

$0.83(0.70-0.92)$

Pooled Sensitivity $=0.70(0.66$ to 0.74$)$

Chi-square $=45.94 ; \mathrm{df}=12(\mathrm{p}=0.0000)$

Inconsistency (l-square) $=73.9 \%$

Fig. 3 Forest plots of sensitivity of synovial fluid PCR for PJ diagnosis

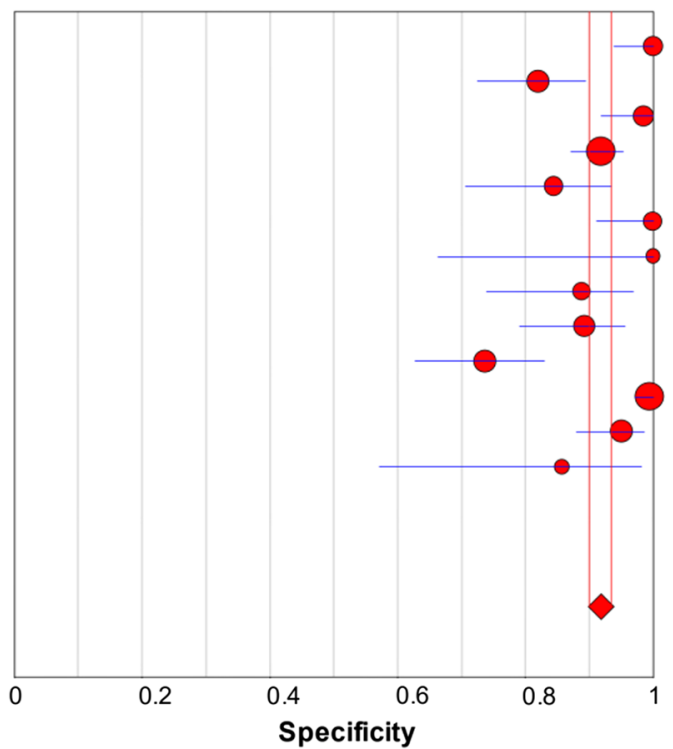

Bergin

Fink

Larsen

Melendez

Melendez

Sebastian

Janz

Janz

Morgenstern

Panousis

Kuo

Melendez

Huang

\section{Specificity $(95 \% \mathrm{Cl})$}

$1.00 \quad(0.94-1.00)$

$0.82 \quad(0.72-0.89)$

$0.98 \quad(0.92-1.00)$

$0.92 \quad(0.87-0.95)$

$0.84 \quad(0.71-0.94)$

$1.00 \quad(0.91-1.00)$

$1.00 \quad(0.66-1.00)$

$0.89 \quad(0.74-0.97)$

$0.89 \quad(0.79-0.96)$

$0.74 \quad(0.63-0.83)$

$0.99 \quad(0.97-1.00)$

$0.95 \quad(0.88-0.99)$

$0.86 \quad(0.57-0.98)$

Pooled Specificity $=0.92(0.90$ to 0.93$)$

Chi-square $=85.94 ; \mathrm{df}=12(\mathrm{p}=0.0000)$

Inconsistency (1-square) $=86.0 \%$

Fig. 4 Forest plots of specificity of synovial fluid PCR for PJI diagnosis

specificity of synovial fluid ( $84 \%$ and $89 \%$, respectively), tissue samples (95\% and $81 \%$, respectively), and sonicate fluid ( $81 \%$ and $96 \%$, respectively) [16], with moderate sensitivity and specificity levels observed for the three sample types. Interestingly, similar results were also reported by the retrospective study performed by Huang and colleagues [17]. In 2018, Jun et al. [15] performed a diagnostic meta-analysis of PCR in PJI, reporting a sensitivity of 0.76 (95\% CI $0.65-0.85)$ and specificity of 0.94 (95\% CI 0.92-0.95). Unfortunately, the pooled result combines samples from synovial fluid, sonicate fluid, and intraoperative tissue. Hence, the diagnostic value of synovial fluid PCR in periprosthetic hip and knee by meta-analysis remained unexplored. The present study is 


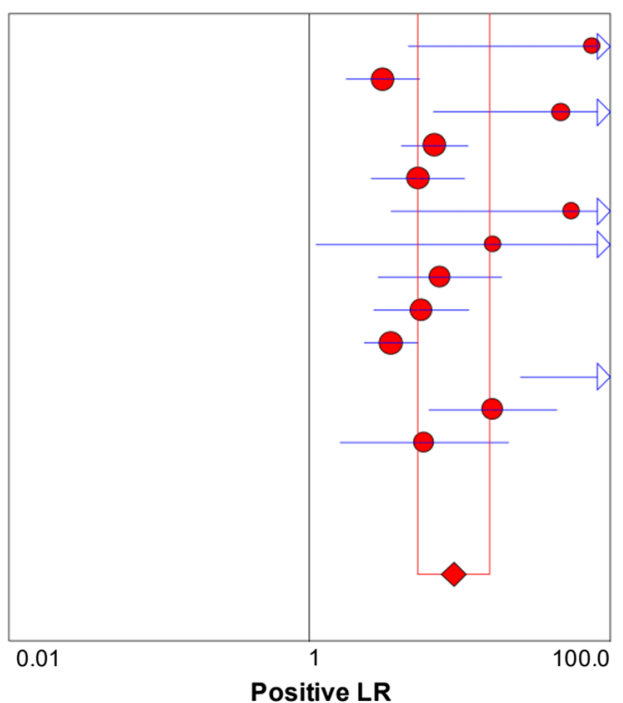

Bergin
Fink
Larsen
Melendez
Melendez
Sebastian
Janz
Janz
Morgenstern
Panousis
Kuo
Melendez
Huang

Random Effects Model

Pooled Positive LR $=9.09$ (5.28 to 15.67)

Cochran- $Q=53.02 ; d f=12(p=0.0000)$

Inconsistency (I-square) $=77.4 \%$

Tau-squared $=0.6135$

Fig. 5 Forest plots of positive likelihood ratio of synovial fluid PCR for PJI diagnosis

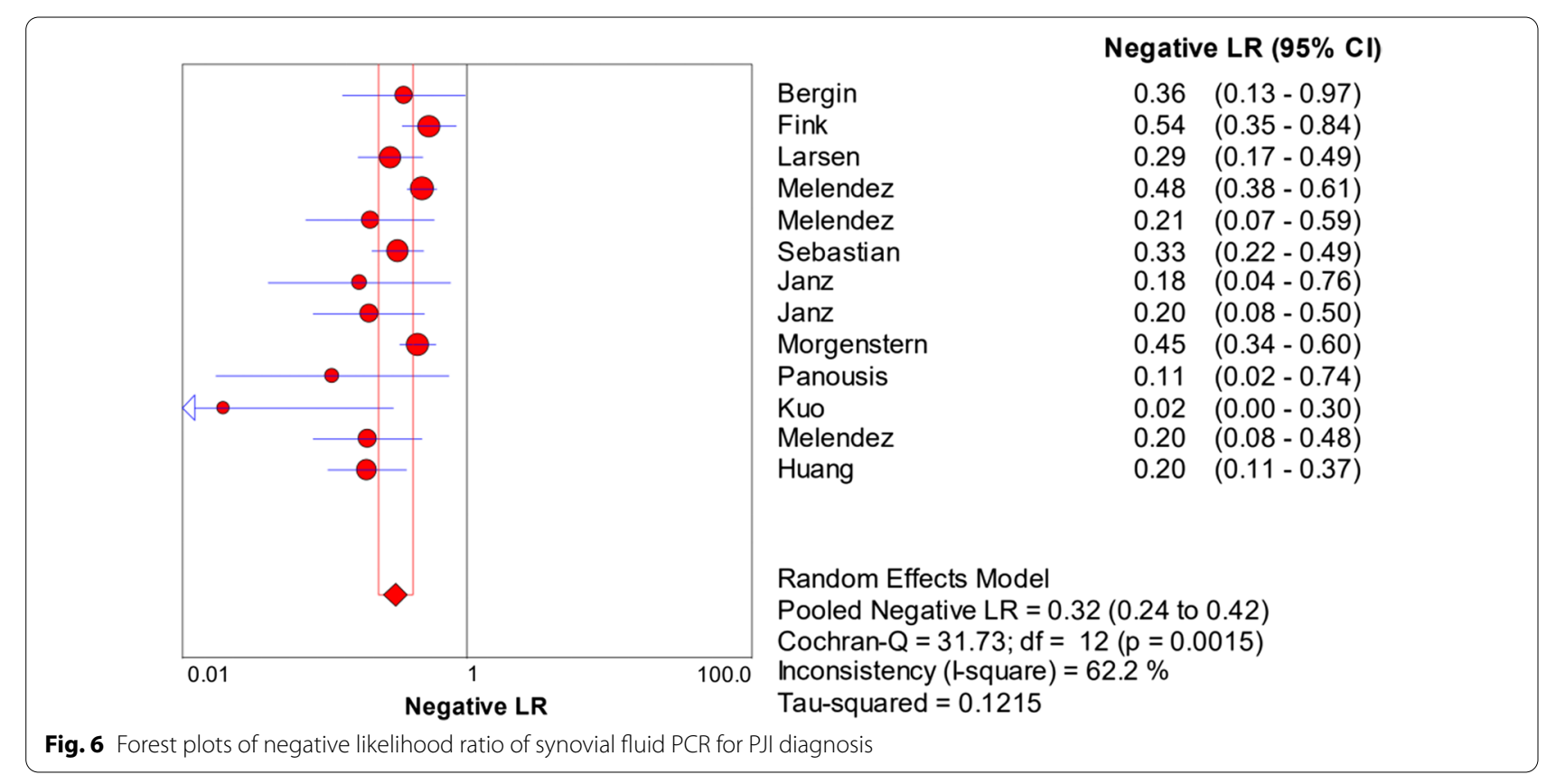

Positive LR $(95 \% \mathrm{Cl})$

$75.86 \quad(4.55-1,265.96)$

$3.09(1.77-5.40)$

$47.14 \quad(6.66-333.45)$

$6.82(4.12-11.30)$

$5.29(2.59-10.83)$

$55.52(3.51-878.78)$

$16.67(1.10-252.34)$

$7.36 \quad(2.86-18.94)$

$5.55 \quad(2.69-11.44)$

$3.49(2.33-5.24)$

$124.23 \quad(25.21-612.18)$

$16.60(6.24-44.13)$

$5.81(1.60-21.09)$ the first meta-analysis evaluating the diagnostic value of synovial fluid PCR for diagnosing infection after hip and knee replacement.

In recent years, the use of joint fluid for diagnosing PJI was a topic of considerable interest. Multiple synovial fluid tests were applied in clinical practice, with several valuable tests incorporated into the new definition of MSIS [10]. Through a literature review of the published meta-analysis of these synovial fluid methods from the
MSIS guideline [26-30], the pooled sensitivity of these tests is superior to that of synovial fluid PCR. The specificity of synovial fluid PCR exceeded that of synovial fluid interleukin-6 (IL-6), CRP, WBC, and PMN, but was lower than synovial fluid culture, alpha-defensins, and LE. Based on the AUC value of meta-analysis of synovial fluid [31], only LE demonstrated excellent accuracy in the diagnosis of PJI, followed by alpha-defensins, IL-6, CRP, and PMN. Furthermore, synovial fluid WBC as well 


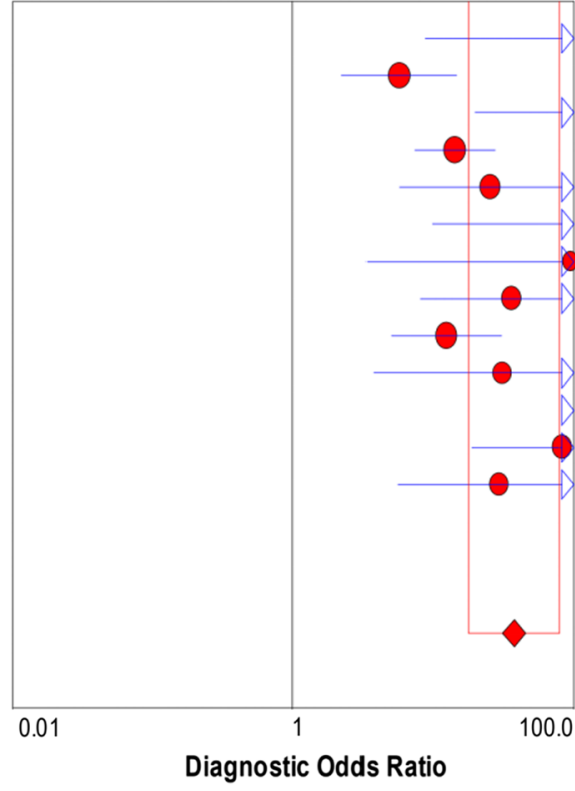

Bergin

Fink

Larsen

Melendez

Melendez

Sebastian

Janz

Janz

Morgenstern

Panousis

Kuo

Melendez

Huang

Random Effects Model

Pooled Diagnostic Odds Ratio $=37.40$ (17.77 to 78.74)

Cochran- $Q=35.74 ; d f=12(p=0.0004)$

Inconsistency (I-square) $=66.4 \%$

Tau-squared $=1.0318$
Diagnostic OR $(95 \% \mathrm{Cl})$

$210.60 \quad(8.72-5,085.37)$

$5.70 \quad(2.25-14.49)$

$162.50 \quad(19.77-1,335.99)$

$14.13(7.29-27.40)$

$25.33 \quad(5.74-111.83)$

$169.84 \quad(9.79-2,947.15)$

$95.00 \quad(3.36-2,682.79)$

$36.00(8.02-161.57)$

$12.29 \quad(4.96-30.45)$

$30.90 \quad(3.76-254.10)$

$6,409.00 \quad(254.23-161,568.28)$

$82.88 \quad(18.83-364.75)$

$29.33(5.58-154.25)$

Fig. 7 Forest plots of diagnostic odds ratio of synovial fluid PCR for PJ diagnosis

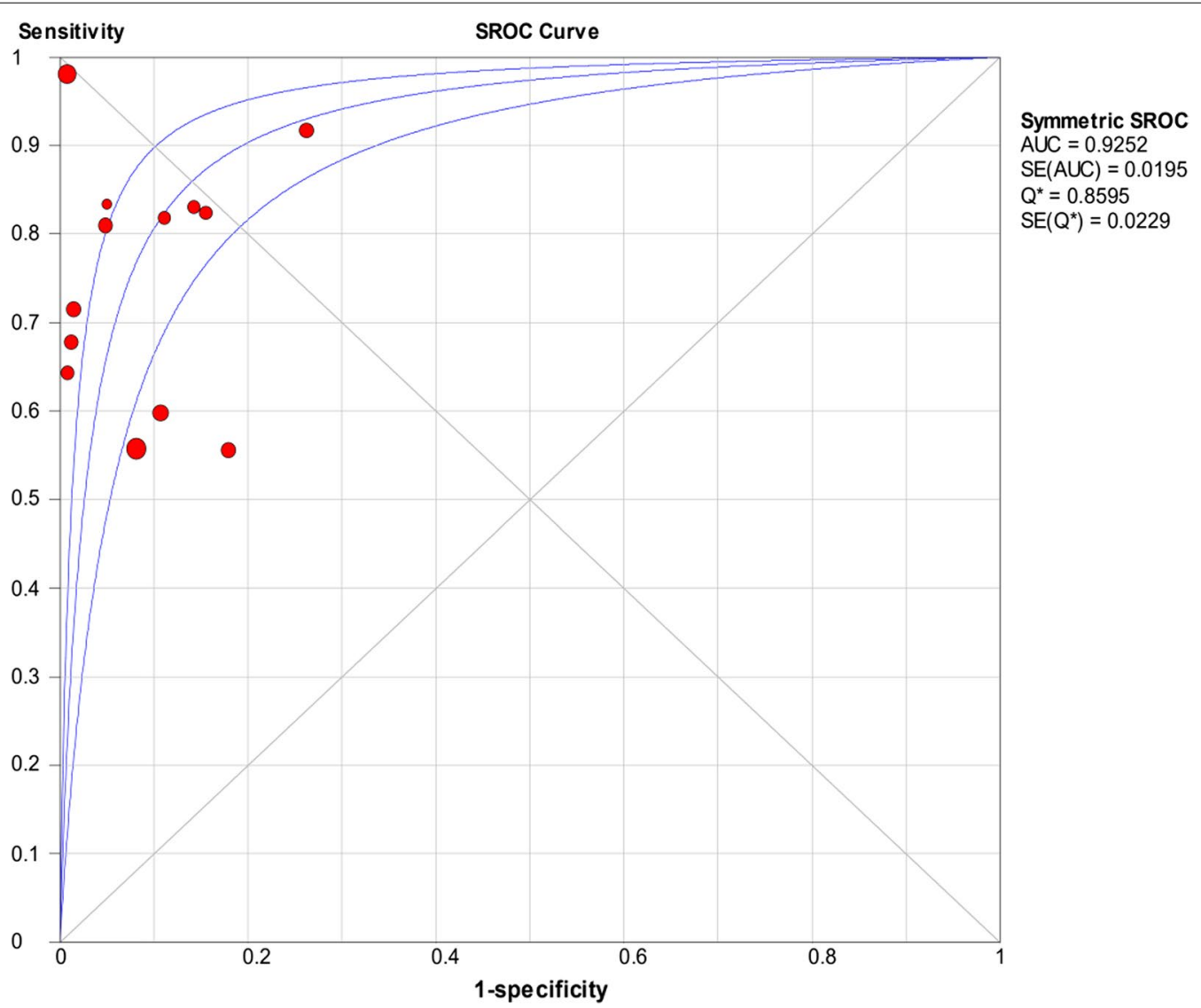

Fig. 8 Summary of SROC of synovial fluid PCR for PJ diagnosis 
Table 2 Comparison of synovial fluid PCR with conventional diagnostic methods from the included studies

\begin{tabular}{|c|c|c|c|c|c|c|c|}
\hline $\begin{array}{l}\text { Diagnostic } \\
\text { method }\end{array}$ & $\begin{array}{l}\text { Number } \\
\text { of } \\
\text { studies }\end{array}$ & $\begin{array}{l}\text { Sensitivity }(95 \% \\
\mathrm{Cl})\end{array}$ & $\begin{array}{l}\text { Specificity (95\% } \\
\text { CI) }\end{array}$ & PLR $(95 \% \mathrm{Cl})$ & NLR $(95 \% \mathrm{Cl})$ & DOR $(95 \% \mathrm{Cl})$ & SROC (SE) \\
\hline CRP & 4 & $0.67(0.54-0.78)$ & $0.79(0.74-0.83)$ & $2.99(2.01-4.45)$ & $0.49(0.26-0.92)$ & 7.55 (3.58-15.94) & $0.7969(0.0428)$ \\
\hline Synovial fluid PCR & 4 & $0.79(0.67-0.87)$ & $0.91(0.88-0.93)$ & $12.05(2.83-51.30)$ & $0.20(0.05-0.89)$ & $93.69(5.10-1721.56)$ & $0.9613(0.0427)$ \\
\hline ESR & 3 & $0.80(0.67-0.90)$ & $0.68(0.63-0.73)$ & $2.58(2.10-3.16)$ & $3.30(0.17-0.52)$ & $10.16(4.72-21.85)$ & $0.8284(0.0391)$ \\
\hline Synovial fluid PCR & 3 & $0.93(0.81-0.99)$ & $0.93(0.90-0.96)$ & $29.92(0.53-1678.60)$ & $0.12(0.01-0.93)$ & $\begin{array}{l}291.64(12.63- \\
6735.22)\end{array}$ & $0.9838(0.0205)$ \\
\hline $\begin{array}{l}\text { Synovial fluid } \\
\text { culture }\end{array}$ & 9 & $0.70(0.65-0.75)$ & $0.98(0.96-0.99)$ & $27.26(16.94-43.85)$ & $0.28(0.20-0.40)$ & $\begin{array}{l}115.11(61.80- \\
214.41)\end{array}$ & $0.9668(0.0079)$ \\
\hline Synovial fluid PCR & 10 & $0.69(0.64-0.74)$ & $0.91(0.89-0.93)$ & $8.21(4.56-14.77)$ & $0.34(0.25-0.46)$ & $32.23(13.94-74.50)$ & ) 0.9173 (0.0239) \\
\hline Tissue culture & 5 & $0.70(0.63-0.75)$ & $0.92(0.89-0.95)$ & $13.10(5.51-31.10)$ & $0.30(0.16-0.54)$ & $41.53(22.40-76.97)$ & ) 0.9305 (0.0152) \\
\hline Synovial fluid PCR & 6 & $0.67(0.61-0.72)$ & $0.88(0.85-0.91)$ & $5.45(3.42-8.68)$ & $0.35(0.26-0.48)$ & $16.93(10.65-26.90)$ & $0.8740(0.0187)$ \\
\hline Histology & 2 & $0.75(0.63-0.85)$ & $1.00(0.96-1.00)$ & $66.59(9.47-468.05)$ & $0.22(0.07-0.69)$ & $\begin{array}{l}325.27(24.60- \\
4300.37)\end{array}$ & NA \\
\hline Synovial fluid PCR & 2 & $0.64(0.53-0.74)$ & $0.81(0.73-0.87)$ & $4.17(2.28-7.62)$ & $0.29(0.06-1.29)$ & $14.20(6.17-32.66)$ & NA \\
\hline $\begin{array}{l}\text { Sonicate fluid } \\
\text { culture }\end{array}$ & 3 & $0.75(0.67-0.82)$ & $0.96(0.90-0.99)$ & $11.97(2.89-49.52)$ & $0.26(0.13-0.53)$ & 50.95 (9.26-280.39) & ) 0.9397 (0.0371) \\
\hline Synovial fluid PCR & 4 & $0.65(0.59-0.71)$ & $0.90(0.86-0.93)$ & $6.06(4.29-8.57)$ & $0.37(0.26-0.53)$ & $15.37(9.49-24.87)$ & $0.8662(0.0203)$ \\
\hline
\end{tabular}

$\mathrm{Cl}$, confidential interval; CRP, C-reactive protein; ESR, erythrocyte sedimentation rate; PCR, polymerase chain reaction; PLR, positive likelihood ratio; NA, not available; NLR, negative likelihood ratio; DOR, diagnostic odds ratio; SROC, Summary receiver operating characteristic; SE, standard error

Table 3 Subgroup analysis of synovial fluid PCR

\begin{tabular}{|c|c|c|c|c|c|}
\hline \multirow[t]{2}{*}{ Variable ( $P$ value) } & \multirow[t]{2}{*}{ Number of studies } & \multicolumn{2}{|c|}{ Sensitivity $(95 \% \mathrm{Cl})$} & \multicolumn{2}{|c|}{ Specificity $(95 \% \mathrm{Cl})$} \\
\hline & & Adjusted & $P$ value & Adjusted & $P$ value \\
\hline \multicolumn{6}{|l|}{ Reference standard $(P=0.41)$} \\
\hline Diagnostic standard & 9 & $0.73[0.64-0.83]$ & $P=0.04$ & $0.95[0.91-0.99]$ & $P=0.91$ \\
\hline Others & 4 & $0.85[0.72-0.98]$ & & $0.94[0.85-1.00]$ & \\
\hline \multicolumn{6}{|l|}{ Acquisition time $(P<0.001)$} \\
\hline Preoperative & 9 & $0.75[0.63-0.86]$ & $P=0.57$ & $0.96[0.91-1.00]$ & $P=0.26$ \\
\hline Intraoperative & 2 & $0.77[0.54-0.99]$ & & $0.93[0.77-1.00]$ & \\
\hline \multicolumn{6}{|l|}{ Number of patients $(P=0.34)$} \\
\hline Number of patients (> 100) & 6 & $0.71[0.59-0.83]$ & $P=0.01$ & $0.95[0.91-1.00]$ & $P=0.72$ \\
\hline Number of patients (<100) & 7 & $0.81[0.71-0.90]$ & & $0.93[0.87-1.00]$ & \\
\hline \multicolumn{6}{|l|}{ Antibiotic treatment $(P<0.001)$} \\
\hline Antibiotic treatment (yes) & 5 & $0.73[0.56-0.89]$ & $P=0.21$ & $0.96[0.91-1.00]$ & $P=0.76$ \\
\hline Antibiotic treatment (no) & 5 & $0.80[0.66-0.94]$ & & $0.91[0.82-1.00]$ & \\
\hline \multicolumn{6}{|l|}{ Target gene $(P=0.1)$} \\
\hline 16S rRNA & 4 & $0.75[0.59-0.90]$ & $P=0.22$ & $0.99[0.96-1.00]$ & $P=0.14$ \\
\hline Others & 9 & $0.77[0.67-0.88]$ & & $0.92[0.86-0.97]$ & \\
\hline
\end{tabular}

as PCR showed good accuracy (Table 4). Although the results of most synovial fluid biomarkers were superior to that of the synovial fluid PCR [26-30], synovial fluid culture and some PCR tests can detect bacteria and could provide a more valuable reference for further comparisons with intraoperative diagnostic results. However, the diagnostic value between synovial fluid culture and PCR for PJI detection is controversial.
Synovial fluid PCR was compared with preoperative and intraoperative tests from the included studies The PCR test was found to have better sensitivity and specificity than that of serum CRP and ESR. In contrast, lower sensitivity and specificity were observed in comparison to all intraoperative methods. Compared with synovial fluid culture, synovial fluid PCR had an almost identical level of sensitivity with synovial fluid culture (69\% vs. 


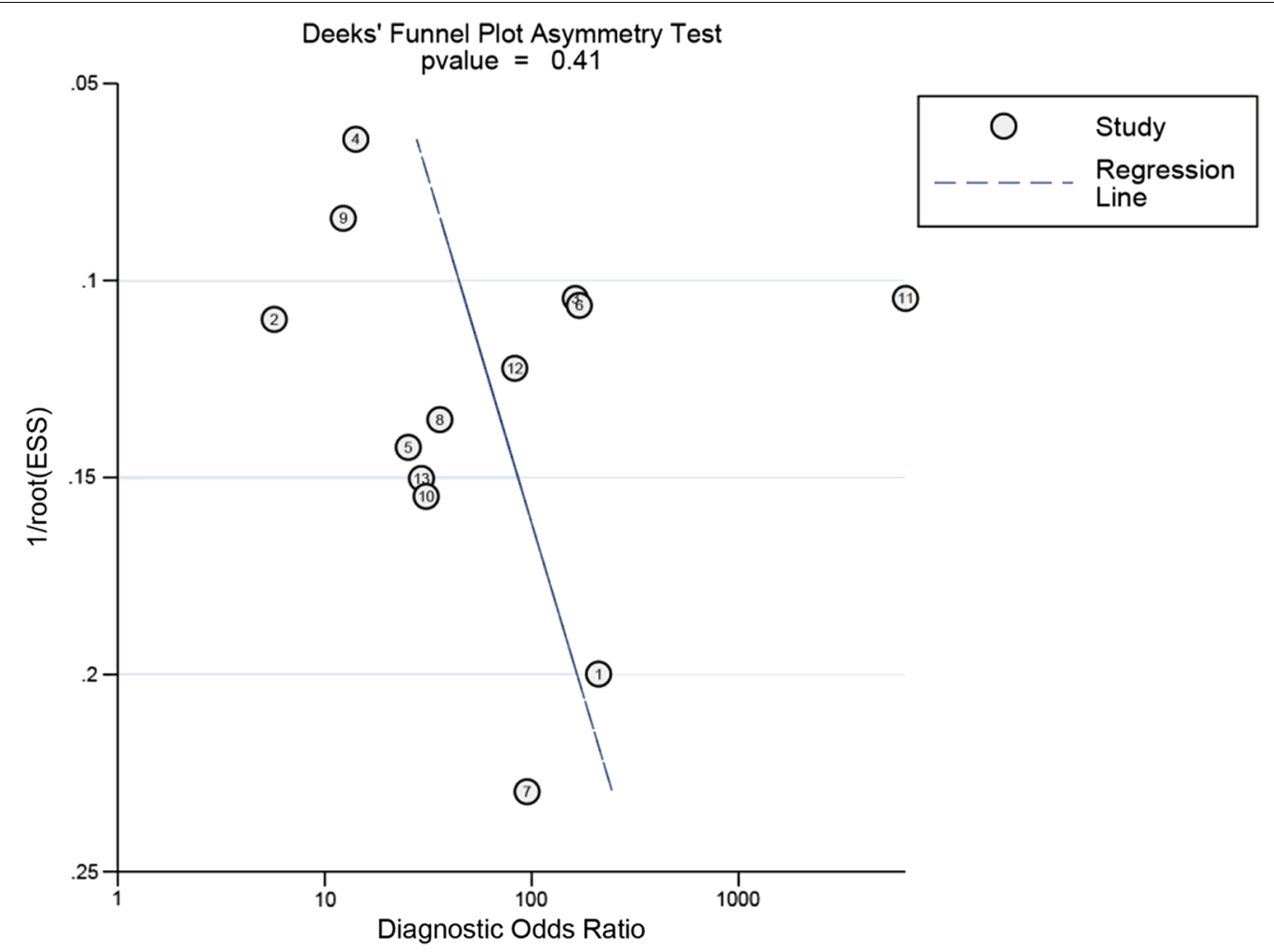

Fig. 9 Deeks'funnel plot for evaluation of publication bias

Table 4 Comparison of synovial fluid PCR with synovial fluid biomarkers using the MSIS definition based meta-analysis results

\begin{tabular}{|c|c|c|c|c|c|c|c|}
\hline References & Test & Location & $\begin{array}{l}\text { Number } \\
\text { of } \\
\text { studies }\end{array}$ & Sensitivity $(95 \% \mathrm{CI})$ & Specificity $(95 \% \mathrm{CI})$ & DOR $(95 \% \mathrm{Cl})$ & AUC \\
\hline [26] & $\begin{array}{l}\text { Synovial fluid alpha- } \\
\text { defensin }\end{array}$ & Hip, knee, shoulder & 16 & $0.87(0.83-0.90)$ & 0.97 (0.96-0.98) & $158.18(74.26-336.91)$ & 0.9685 \\
\hline [26] & Synovial fluid LE & Hip, knee, shoulder & 12 & $0.87(0.84-0.90)$ & $0.96(0.95-0.97)$ & $170.09(97.63-296.32)$ & 0.9818 \\
\hline [27] & Synovial fluid IL-6 & Hip, knee, shoulder & 8 & $0.91(0.82-0.96)$ & $0.90(0.84-0.95)$ & $101(28-358)$ & 0.96 \\
\hline [28] & Synovial fluid CRP & Hip, knee & 6 & $0.92(0.86-0.96)$ & $0.90(0.87-0.93)$ & $101.40(48.07-213.93)$ & 0.9663 \\
\hline [29] & $\begin{array}{l}\text { Synovial fluid WBC } \\
\text { count }\end{array}$ & Hip, knee & 11 & $0.900(0.872-0.922)$ & $0.898(0.814-0.947)$ & $84.4(40.3-157)$ & 0.910 \\
\hline [29] & Synovial fluid PMN & Hip, knee & 11 & $0.906(0.870-0.933)$ & $0.861(0.808-0.901)$ & $62.8(32.7-109)$ & 0.940 \\
\hline [30] & Synovial fluid culture & Hip, knee & 34 & $0.72(0.65-0.78)$ & $0.95(0.93-0.97)$ & $52(31-86)$ & 0.94 \\
\hline Current study & Synovial fluid PCR & Hip, knee & 11 & $0.70(0.66-0.74)$ & $0.92(0.90-0.93)$ & $37.4(17.77-78.74)$ & 0.9252 \\
\hline
\end{tabular}

$\mathrm{Cl}$, confidence interval; AUC, Area under the curve; DOR, Diagnostic odds ratio; CRP, C-reactive protein; IL-6, Interleukin-6; LE, Leukocyte esterase; PCR, Polymerase chain reaction; PMN, Polymorphonuclear leukocytes; WBC, White blood cell

$70 \%$, respectively) and a lower specificity level (91\% vs. 98\%, respectively). Synovial fluid PCR and culture were also observed to have similar results in comparison to the previous meta-analysis of synovial fluid culture, with a sensitivity of $70 \%$ and $72 \%$, respectively, and a specificity of $92 \%$ and $95 \%$, respectively [30]. Although the current meta-analysis and our subgroup results showed that the sensitivity and specificity of synovial fluid PCR were lower than that of synovial fluid, PCR has several advantages in regard to the detection of bacteria. Synovial fluid PCR has been reported to rapidly provide results within 3-72 h [14, 23, 25], and could also detect culture-negative bacteria $[11,12,21,22,25]$. Sujeesh and co-workers reported that the sensitivity of $16 \mathrm{~S}$ rRNA 
PCR and synovial fluid culture was $68.1 \%$ and $70.2 \%$, respectively [22]. PJI was detected by PCR in five cases that were negative by synovial fluid culture. Synovial fluid multiplex PCR identified 12 cases negative by synovial fluid culture, with 10 cases caused by low-virulence bacteria (coagulase-negative staphylococci and Cutibacterium acnes) [25]. The author also found that more cases of polymicrobial infections were detected by synovial PCR than synovial fluid culture (four vs. two cases), with similar results also reported by Melendez and colleagues $[18,21]$. Due to rare cases of mixed infection in these studies, further research is required. However, in cases treated with antibiotics before specimen collection, the testability of synovial fluid PCR was lower than synovial fluid culture. A comparison of the PCR panel and synovial fluid culture in patients that received antibiotics within 30 days before joint aspiration revealed the sensitivity of the PCR panel and synovial culture to be $64.5 \%$ and $85.4 \%$, respectively [21]. In another study, PCR-ESI/ MS detected eight of nine PJI cases who had received antibiotics within 30 days, whereas synovial fluid culture detected all nine cases [18]. The use of antibiotics before PCR analysis most likely impacts culture results. Meta-regression analysis results from the current study show that the sensitivity level of cases receiving antibiotic therapy were less than cases without antibiotic therapy (73\% vs. $80 \%$ ). Moreover, meta-regression was also analyzed in the preoperative and intraoperative aspiration groups, with slightly higher sensitivity and lower specificity observed for the intraoperative test compared to the preoperative test (sensitivity: $77 \%$ vs. $75 \%$, respectively; specificity: $93 \%$ vs. $96 \%$ ). In contrast, the meta-analysis of synovial fluid white cell count performed by $\mathrm{Qu}$ and co-workers [32] found that preoperative collection had a higher sensitivity than intraoperative samples (91\% vs. $77 \%$, respectively), and lower specificity than that of intraoperative samples ( $89 \%$ vs. $97 \%$ ). However, due to the limited data of the studies included from our intraoperative study (two studies) and different tests performed in these two meta-analyses, whether intraoperative and preoperative sample collection infers with the diagnostic accuracy remains an avenue for further exploration.

Although various types of synovial fluid PCR have been tested in the clinical diagnosis of PJI, the diagnostic ability has most likely been disregarded. The most frequently described disadvantage of PCR is FP results, with the potential impacting factors, including the use of different target genes, PCR type, laboratory technician skills, and laboratory conditions [13, 19, 22, 24]. The use of $16 \mathrm{~S} / 28 \mathrm{~S}$ rRNA RT-PCR with high-quality control standards demonstrated excellent results, with a sensitivity of $100 \%$ and specificity of $99.5 \%$ [13].Sebastian and colleagues [22] found that DNase treatment could reduce exogenous bacterial contamination, with no FP results observed in synovial fluid PCR: however, the sensitivity was affected. Further studies are required to determine the most suitable type of PCR for PJI diagnosis and the standard procedure required.

The present study has several limitations. First, the identified studies used different types of PCR; therefore, the overall result may impact the estimates of diagnostic accuracy. Second, meta-regression analysis was not performed in regard to the prosthesis type or sample condition to further explore sources of heterogeneity. The type of prosthesis described in the included studies were the knee or both the hip and knee; however, studies focusing only on the periprosthetic hip were not found. Therefore, further analysis of differences between the hip and knee could not be performed in meta-regression analysis. Regarding the sample condition, frozen specimens were used in the studies; however, there was ambiguity in terms of the use of fresh samples. Third, the diagnostic accuracy of synovial fluid PCR may be affected by the standard definition of PJI [33]. Fourth, the current metaanalysis only included the English article, which was published in the database of Web of Science, PubMed, and Scopus. However, the relevant published literature from other languages or other databases is probably lacking.

\section{Conclusions}

The diagnostic capability of synovial fluid PCR is not superior to that of synovial fluid culture. However, in cases of negative synovial fluid culture with highly suspected early-stage infection, synovial fluid PCR can be used as a rapid diagnostic confirmatory tool.

\section{Abbreviations}

AUC: Area under the curve; C: Clinical signs of infection; $\mathrm{Cl}$ : Confidence interval; CRP: C-reactive protein; DOR: Diagnostic odds ratio; EBJIS: European Bone and Joint Infection Society; ESI/MS: Electrospray ionization mass spectrometry; ESR: Erythrocyte sedimentation rate; FN: False-negative; FP: False-positive; $\mathrm{H}$ : Histological examination; IDSA: Infectious Diseases Society of America; ICM: International Consensus Meeting; IL-6: Interleukin-6; LE: Leukocyte esterase; M: Microbiological or laboratory examination; MeSH: Medical subject headings; MSIS: Musculoskeletal Infection Society; NLR: Negative likelihood ratio; N: No; NA: Not available; P: Presence sinus tract or purulence around the prosthesis; PCR: Polymerase chain reaction; PJI: Periprosthetic joint infection; PLR: Positive likelihood ratio; PMN: Polymorphonuclear; QUADAS-2: Quality Assessment of Diagnostic Accuracy Studies-2; qPCR: Quantitative PCR; R: Radiographic images; RT: Real-time; SROC: Summary receiver operating characteristic; SE: Standard error; Sen: Sensitivity; Spe: Specificity; TN: True negative; TP: True positive; WBC: White blood cell count; Y:Yes.

\section{Acknowledgements}

We acknowledge support from the German Research Foundation (DFG) and the Open Access Publication Fund of Charité-Universitätsmedizin Berlin.

\section{Authors' contributions}

LC conceived the design of the study. LC, LH and YX performed and collected the data and contributed to the design of the study. ZFZ and XC analyzed the 
data. LC prepared and revised the manuscript. AT edited and reviewed the manuscript. All authors read and approved the final manuscript.

\section{Funding}

Open Access funding enabled and organized by Projekt DEAL. German Research Foundation (DFG) and the Open Access Publication Fund of Charité-Universitätsmedizin Berlin.

\section{Availability of data and materials \\ Not applicable.}

\section{Declarations}

\section{Ethics approval and consent to participate}

Not applicable.

\section{Consent for publication}

Not applicable.

\section{Competing interests}

The authors declare that they have no competing interests.

\section{Author details}

${ }^{1}$ Department of Orthopaedic Surgery, Beijing Jishuitan Hospital, Beijing, China. ${ }^{2}$ Center for Musculoskeletal Surgery (CMSC), Charité-Universitätsmedizin Berlin, Berlin, Germany. ${ }^{3}$ Department of Orthopaedic Surgery, General Hospital of People's Liberation Army, Beijing, China.

Received: 26 May 2021 Accepted: 28 October 2021

Published online: 04 January 2022

\section{References}

1. Delanois RE, Mistry JB, Gwam CU, Mohamed NS, Choksi US, Mont MA Current epidemiology of revision total knee arthroplasty in the United States. J Arthroplasty. 2017;32:2663-8.

2. Gwam CU, Mistry JB, Mohamed NS, Thomas M, Bigart KC, Mont MA, et al. Current epidemiology of revision total hip arthroplasty in the United States: National Inpatient Sample 2009 to 2013. J Arthroplasty. 2017;32:2088-92.

3. Kurtz SM, Lau E, Watson H, Schmier JK, Parvizi J. Economic burden of periprosthetic joint infection in the United States. J Arthroplasty. 2012;27:61-5.e1.

4. Natsuhara KM, Shelton TJ, Meehan JP, Lum ZC. Mortality during total hip periprosthetic joint infection. J Arthroplasty. 2019:34:S337-342.

5. Lum ZC, Natsuhara KM, Shelton TJ, Giordani M, Pereira GC, Meehan JP. Mortality during total knee periprosthetic joint infection. J Arthroplasty. 2018;33:3783-8.

6. Li C, Ojeda-Thies C, Xu C, Trampuz A. Meta-analysis in periprosthetic joint infection: a global bibliometric analysis. J Orthop Surg Res. 2020;15:251.

7. Li C, Renz N, Trampuz A. Management of periprosthetic joint infection. Hip Pelvis. 2018;30:138-46.

8. Gollwitzer H, Dombrowski Y, Prodinger PM, Peric M, Summer B, Hapfelmeier A, et al. Antimicrobial peptides and proinflammatory cytokines in periprosthetic joint infection. J Bone Joint Surg Am. 2013;95:644-51.

9. Karbysheva S, Yermak K, Grigoricheva L, Renz N, Perka C, Trampuz A. Synovial fluid d-lactate-A novel pathogen-specific biomarker for the diagnosis of periprosthetic joint infection. J Arthroplasty. 2020;35(8):2223-9.

10. Parvizi J, Tan TL, Goswami K, Higuera C, Della Valle C, Chen AF, et al. The 2018 definition of periprosthetic hip and knee infection: an evidencebased and validated criteria. J Arthroplasty. 2018;33:1309-14.e2.

11. Chenouard R, Hoppé E, Lemarié C, Talha A, Ducellier F, Ferchaud F, et al. A rare case of prosthetic joint infection associated with Coxiella burnetii. Int J Infect Dis. 2019:87:166-9.
12. Farrell JJ, Larson JA, Akeson JW, Lowery KS, Rounds MA, Sampath R, et al. Ureaplasma parvum prosthetic joint infection detected by PCR. J Clin Microbiol. 2014;52:2248-50.

13. Kuo F-C, Lu Y-D, Wu C-T, You H-L, Lee G-B, Lee MS. Comparison of molecular diagnosis with serum markers and synovial fluid analysis in patients with prosthetic joint infection. Bone Joint J. 2018;100-B:1345-51.

14. Fink B, Steurer M, Hofäcker S, Schäfer P, Sandow D, Schuster P, et al. Preoperative PCR analysis of synovial fluid has limited value for the diagnosis of periprosthetic joint infections of total knee arthroplasties. Arch Orthop Trauma Surg. 2018;138:871-8.

15. Jun $Y$, Jianghua L. Diagnosis of periprosthetic joint infection using polymerase chain reaction: an updated systematic review and metaanalysis. Surg Infect. 2018;19:555-65.

16. Qu X, Zhai Z, Li H, Li H, Liu X, Zhu Z, et al. PCR-based diagnosis of prosthetic joint infection. J Clin Microbiol. 2013;51:2742-6.

17. Huang Z, Wu Q, Fang X, Li W, Zhang C, Zeng H, et al. Comparison of culture and broad-range polymerase chain reaction methods for diagnosing periprosthetic joint infection: analysis of joint fluid, periprosthetic tissue, and sonicated fluid. Int Orthop. 2018;42:2035-40.

18. Melendez DP, Uhl JR, Greenwood-Quaintance KE, Hanssen AD, Sampath R, Patel R. Detection of prosthetic joint infection by use of PCRelectrospray ionization mass spectrometry applied to synovial fluid. J Clin Microbiol. 2014;52(6):2202-5.

19. Bergin PF, Doppelt JD, Hamilton WG, Mirick GE, Jones AE, Sritulanondha $\mathrm{S}$, et al. Detection of periprosthetic infections with use of ribosomal RNA-based polymerase chain reaction. J Bone Joint Surg Am. 2010;92:654-63.

20. Larsen LH, Khalid V, Xu Y, Thomsen TR, Schønheyder HC. Differential contributions of specimen types, culturing, and $16 \mathrm{~S}$ rRNA sequencing in diagnosis of prosthetic joint infections. J Clin Microbiol. 2018;56(5):01351-417.

21. Melendez DP, Greenwood-Quaintance KE, Berbari EF, Osmon DR, Mandrekar JN, Hanssen AD, et al. Evaluation of a genus- and group-specific rapid PCR assay panel on synovial fluid for diagnosis of prosthetic knee infection. J Clin Microbiol. 2016;54:120-6.

22. Sebastian S, Malhotra R, Sreenivas V, Kapil A, Chaudhry R, Dhawan B. Utility of 165 rRNA PCR in the synovial fluid for the diagnosis of prosthetic joint infection. Ann Lab Med. 2018;38:610-2.

23. Janz V, Schoon J, Morgenstern C, Preininger B, Reinke S, Duda G, et al. Rapid detection of periprosthetic joint infection using a combination of $16 \mathrm{~s}$ rDNA polymerase chain reaction and lateral flow immunoassay: a pilot study. Bone Joint Res. 2018;7:12-9.

24. Panousis K, Grigoris P, Butcher I, Rana B, Reilly JH, Hamblen DL. Poor predictive value of broad-range PCR for the detection of arthroplasty infection in 92 cases. Acta Orthop. 2005;76:341-6.

25. Morgenstern C, Cabric S, Perka C, Trampuz A, Renz N. Synovial fluid multiplex PCR is superior to culture for detection of low-virulent pathogens causing periprosthetic joint infection. Diagn Microbiol Infect Dis. 2018;90:115-9.

26. Chen Y, Kang X, Tao J, Zhang Y, Ying C, Lin W. Reliability of synovial fluid alpha-defensin and leukocyte esterase in diagnosing periprosthetic joint infection (PJI): a systematic review and meta-analysis. J Orthop Surg Res. 2019;14:453.

27. Xie K, Dai K, Qu X, Yan M. Serum and synovial fluid interleukin-6 for the diagnosis of periprosthetic joint infection. Sci Rep. 2017;7:1496.

28. Wang C, Wang Q, Li R, Duan J-Y, Wang C-B. Synovial fluid C-reactive protein as a diagnostic marker for periprosthetic joint infection: a systematic review and meta-analysis. Chin Med J. 2016;129:1987-93.

29. De Fine M, Giavaresi G, Fini M, Illuminati A, Terrando S, Pignatti G. The role of synovial fluid analysis in the detection of periprosthetic hip and knee infections: a systematic review and meta-analysis. Int Orthop. 2018;42:983-94.

30. Qu X, Zhai Z, Wu C, Jin F, Li H, Wang L, et al. Preoperative aspiration culture for preoperative diagnosis of infection in total hip or knee arthroplasty. J Clin Microbiol. 2013;51:3830-4.

31. Jones CM, Athanasiou T. Summary receiver operating characteristic curve analysis techniques in the evaluation of diagnostic tests. Ann Thorac Surg. 2005;79(1):16-20. 
32. Qu X, Zhai Z, Liu X, Li H, Wu C, Li Y, et al. Evaluation of white cell count and differential in synovial fluid for diagnosing infections after total hip or knee arthroplasty. PLoS ONE. 2014;9:e84751.

33. Li C, Renz N, Trampuz A, Ojeda-Thies C. Twenty common errors in the diagnosis and treatment of periprosthetic joint infection. Int Orthop. 2020;44(1):3-14.

\section{Publisher's Note}

Springer Nature remains neutral with regard to jurisdictional claims in published maps and institutional affiliations.

- fast, convenient online submission

- thorough peer review by experienced researchers in your field

- rapid publication on acceptance

- support for research data, including large and complex data types

- gold Open Access which fosters wider collaboration and increased citations

- maximum visibility for your research: over $100 \mathrm{M}$ website views per year

At BMC, research is always in progress.

Learn more biomedcentral.com/submissions 